\title{
Humanlike robots: state of the art and challenges
}

\section{Yoseph Bar-Cohen}

Yoseph Bar-Cohen, "Humanlike robots: state of the art and challenges," Proc. SPIE 10965, Bioinspiration, Biomimetics, and Bioreplication IX, 1096507 (13 March 2019); doi: 10.1117/12.2513709

Event: SPIE Smart Structures + Nondestructive Evaluation, 2019, Denver, Colorado, United States 


\title{
Humanlike robots - state of the art and challenges
}

\author{
Yoseph Bar-Cohen \\ Jet Propulsion Laboratory (JPL), California Institute of Technology, \\ 4800 Oak Grove Drive, Pasadena, CA 91109-8099, \\ yosi@jpl.nasa.gov, web: http://ndeaa.jpl.nasa.gov
}

Humans have always sought to mimic the appearance, mobility, functionality, intelligent operation, and thinking process of biological creatures. In recent years, significant advances have been made in making sophisticated biologically inspired systems. Using these advances, scientists and engineers are increasingly reverse engineering many animals' capabilities. Progress in artificial intelligence, artificial vision, and many other biomimetic related fields are leading to many benefits to humankind. The mimicking of humans is the ultimate and most challenging goal of developing biologically inspired technologies. Lifelike robots that appear and function like humans are becoming increasingly an engineering reality. The use of biomimetic technologies are enabling the possibility of making realistically looking and operating robots. New actuators, sensors and software control algorithms are being developed to support the mobility and the operation in the vicinity and in cooperation with humans. The accelerated pace of the advances in biomimetics seems to make inevitable the development of such machines as our household appliance or even companion. This paper covers the current state-of-the-art and challenges to making humanlike robots.

\section{Keywords}

Biomimetics, Robotics, Humanlike Robots, Actuators

\section{Introduction}

The human species is the most sophisticated result of nature's trial and error evolution. Our appearance, capabilities and intelligence have always been the subject of mimicking and successes are continually been reported [Dautenhahn and Nehaniv, 2002; Bar-Cohen 2005; Vincent, 2005; Bar-Cohen, 2011]. Life-like robots that resemble humans are being developed using advances in computer science, materials science, real-time imaging and recognition, speech interpretation, biped dynamic control and many other technologies [Bar-Cohen and Breazeal, 2003; Bar-Cohen and Hanson, 2009]. The developed robots are being equipped with impressive capabilities and sophistication, including expressing emotions verbally and facially, and responding emotionally in conversation with natural humans [Breazeal, 2002]. Electroactive polymers (EAP), also known as artificial muscles [Bar-Cohen, 2004], are being used to produce biologically inspired mechanisms that were once considered science fiction. As progress is being made, having such robots present in our home or business is increasingly becoming a potential realistic engineering. Like in nature, the development of humanlike robots has been an evolutionary one and an artistic view of this evolution is shown in Figure 1 [Bar-Cohen and Hanson, 2009].

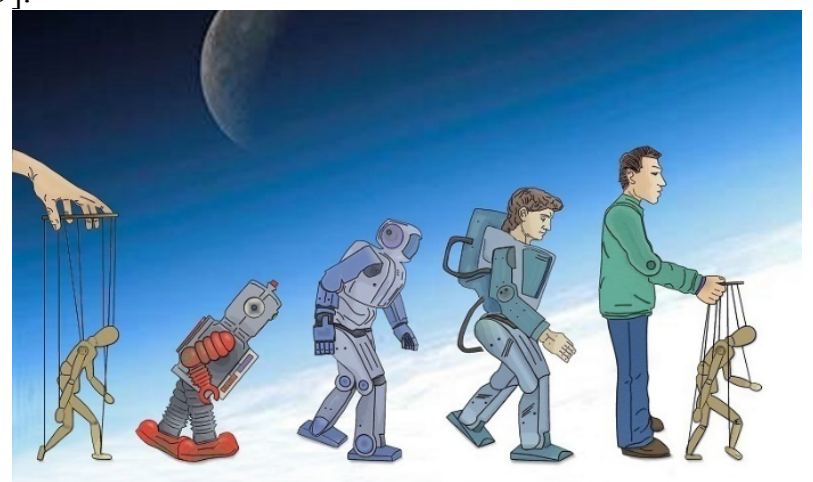

Figure 1: Artistic vision of the evolution of humanlike robots (illustration courtesy of the graphic artist Adi Marom) Bar-Cohen and Hanson, 2009].

To be considered a humanlike robot it is necessary to be similar to humans; thus, the robot needs to have a central torso with a head on top, two arms that are arranged symmetrically on either side, as well as two legs that are symmetrically arranged below the torso to provide upright bipedal posture and mobility. The arms must have hands on their ends and must be equipped with fingers for grasping and manipulating objects, while each leg has to have a foot, 
used for locomotion. Robots with humanlike features are known by several names including humanoids, androids and automatons [Bar-Cohen and Hanson, 2009]. The author distinguishes between the terms 'humanoids' and 'human-like robots' as shown in Figure 2 and Figure 3.

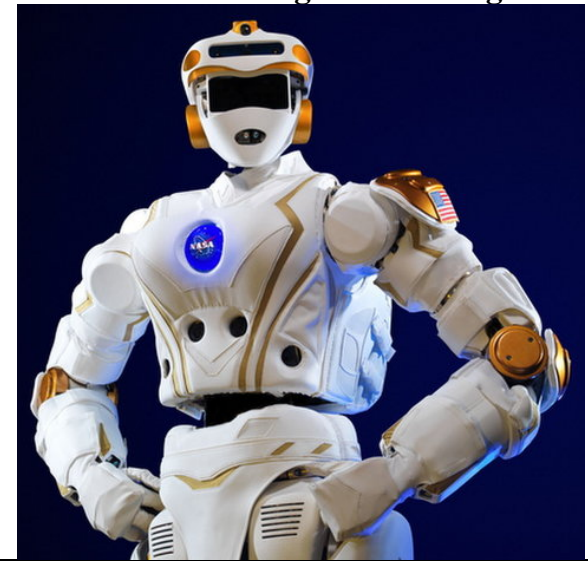

Figure 2: The NASA Johnson Space Center's humanoid Valkyrie robot. Source: NASA-JSC/DARPA.

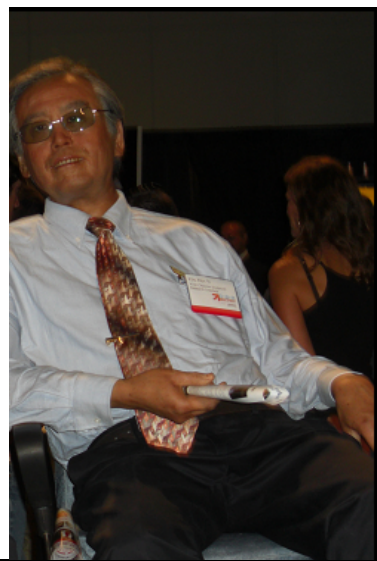

Figure 3: An example of a humanlike robot showing the clone of the roboticist Zou Renti, China. The photo was taken by the author at Wired magazine's NextFest, held on 14 September 2007 in Los Angeles, CA.

The ancient Greeks were the first to envision humanlike machines, where their Hephaestus god was philosophized to have created his own mechanical helpers in the form of young women [Rosheim, 1994]. In a Jewish legend from the $16^{\text {th }}$ century, a servant called the Golem of Prague was brought to life by a rabbi called Loeb. Another famous story about humanlike form is Frankenstein [Shelley, 1818], and it is monster that was created of human body parts and brought to life by the scientist Victor Frankenstein. Common to the golem and Frankenstein stories is the instilling of life into a humanlike form that ultimately ended with disastrous consequences. This commonality in the Western culture suggests that creating humanlike creatures leads to evil results. The first individual who made a sketch of a human-like machine is Leonardo da Vinci, where in 1495 he designed the mechanical knight known as Leonardo's robot [Rosheim, 1996]. As advances in technology were made so the capability of the developed robots, where initially they were operated by mechanical energy stored in a spring while today there are numerous types of actuation mechanisms that are used.

Current humanlike robots are equipped with artificial intelligence making them smart, lifelike machine. While the critical need to survive the risks and dangers of the surrounding environment motivates biological creatures' intelligence, the advances in making lifelike robots is motivated by efforts to make them as good as or more capable than humans. This is achieved by the development of effective microprocessors, autonomous operation algorithms, humanlike materials, dynamic stability and biped mobility, as well as sensors that imitate our senses (seeing, hearing, smelling, etc.). An example of the use of life-like material in a humanlike robotic head is shown in Figure 4, where human skin is imitated using Frubber skin that was developed by David Hanson [Bar-Cohen and Hanson, 2009]. 


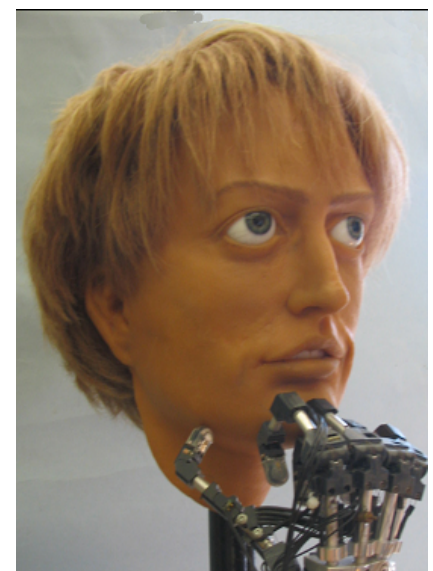

Figure 4: A humanlike robot made with Frubber skin making it lifelike. The head (created by David Hanson) and the hand (created by Graham Whitely) shown in this photo are located in the author's NDEAA lab at JPL.

Operating a robot in the complex and unpredictable natural environments is virtually impossible to pre-program. Therefore, they are incorporated with artificial intelligence (AI) using concepts and methodologies that are inspired and guided by nature [Asimov, 1942; Bar-Cohen and Hanson, 2009]. This allows them to deal with the terrain autonomously as well as learn from their experience and adapt accordingly. The software methodologies are increasingly resemble the organization and functionality of the human central nervous system, helping the robots to perceive, interpret, respond and adapt to their environment.

In general, robots are widely used in industry to perform complex manufacturing tasks including the assembly of automobiles. Other applications include planetary (Figure 5) and deep ocean exploration; operating in areas with toxic gases, radioactivity, dangerous chemicals, bad odors, biological hazards, or extreme temperature environments; cleaning hazardous waste; sweeping mine fields; bomb disposal; search and rescue. Entertainment applications are the most widespread including humanlike robotic toys that are available for purchase in many stores. One indicator for the direction of robotic toy development is the humanlike Einstein robot toy. It is being developed as an interactive learning companion that seeks to become a synthetic friend dedicated to the child for whom it would be purchased (Figure 6).

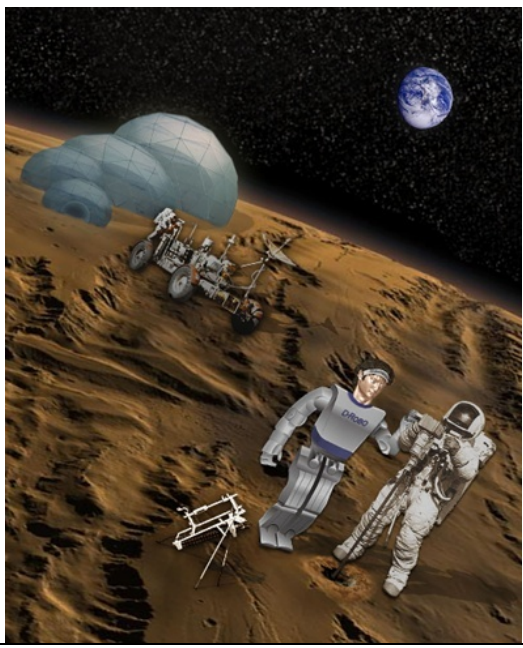

Figure 5: A futuristic vision of the role of human-like robots as human assistants in planetary exploration of the universe (illustration courtesy of graphic artist Adi Marom) [Bar-Cohen and Hanson, 2009].

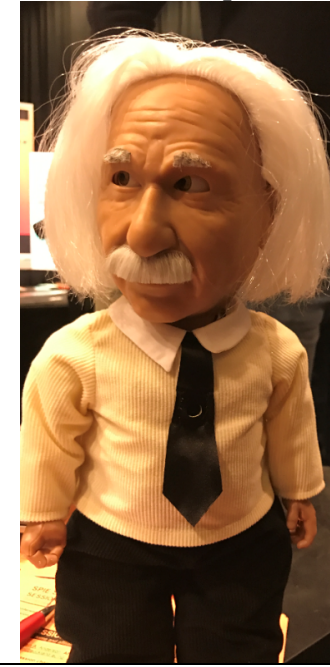

Figure 6: The human-like robot, Einstein toy that is being developed as an interactive learning companion at Hanson Robotics. Photographed by the author at the 2017 SPIE EAP-in-Action Session.

\section{Is it necessary to have robots with humanlike form}

The objective to develop humanlike robots is driven by the fact that the world we built around us has been made to fit our average body size, shape and capabilities [Harris, 2007]. In order to make the most effective assistant, the robot 
needs to match our shape, size and capabilities to do the things we do. This includes reach a door handle, listen to us at eye level, climb stairs, sit on our chair, drive our car and perform many other support tasks. In addition, to simplify the communication it is highly beneficial to have the robot able to speak our language and, since we respond intuitively to body language and gestures, it is essential that the robot is able to make facial and body expressions. Moreover, making humanlike robots is an effective way of making robots more intelligent [Stoica, 2001] and autonomous to allow it to deal with static obstacles as well as negotiate with dynamic objects (e.g., as people, pets and automobiles). The robot may need to be able to walk in a crowded street, cross a street with busy traffic while obeying pedestrian laws, or walk in a complex terrain consisting of unpaved roads and various obstacles. Constructing a life-like robots with humanlike form requires several key technologies including the materials, actuators, sensors, smart controls, mechanisms of mobility and manipulation of the hands and legs, hearing and verbal communication, seeing and image interpretation, the ability to identify and avoid obstacles, as well as determining and addressing risks. The humanlike robot needs to have effective control and artificial intelligence algorithms to interact with its environment and with humans [Plantec and Kurzwell, 2003].

\section{Actuators and artificial muscles}

Actuators emulate muscles and they are used to mobilize robots as well as to move the appendages and other parts. The actuators that are used include electric, pneumatic, hydraulic, piezoelectric, shape-memory alloys and ultrasonic actuators. Electric motors are widely used to move humanlike robots parts, but they behave differently than our natural muscles and have an entirely different operational mechanism. Natural muscles are both compliant and linear in behavior [Full and Meijir, 2004]. Emulating the operation of muscles is important, since they address key control requirements for the life-like operation of robots. The actuators that are the closest to emulating natural muscles are electroactive polymers (EAP) and, for this reason, they are referred to as "artificial muscles" [Bar-Cohen, 2004]. Many of the EAP materials that are known today emerged in the 1990s but remain weak in terms of their ability to perform significant mechanical tasks such as lifting heavy objects.

Recognizing the need for international cooperation, the author initiated and organized in March 1999 the first annual international SPIE EAP Actuators and Devices (EAPAD) Conference [Bar-Cohen, 1999]. At the opening of the first conference, he posed the challenge to scientists and engineers worldwide to develop a robotic arm that is actuated by artificial muscles to be able to win in an armwrestling match against a human opponent (see the icon of the match in Figure 7. In the first contest, an arm wrestling match with a human (a 17-year old high school female student) was held on 7 March 2005. Three robotic arms participated in the contest and the girl won against all of them. The second Artificial Muscles Arm-wrestling Contest was held on 27 February 2006. Rather than wrestling with a human opponent, a custom-made measuring fixture was used to test the EAP-actuated arms for speed and pulling force. To establish a baseline for performance comparison, the capability of the student from the 2005 contest was measured first. It is interesting to note that the 2006 results showed the capability (force and speed) of the EAP actuators to be two orders of magnitude lower than that of the student. The current issue is that the available EAP actuators are still significantly weaker in force and operation speed compared to humans. Once sufficient advances have been made in EAP capability, a professional wrestler will be invited for a human/machine wrestling match.

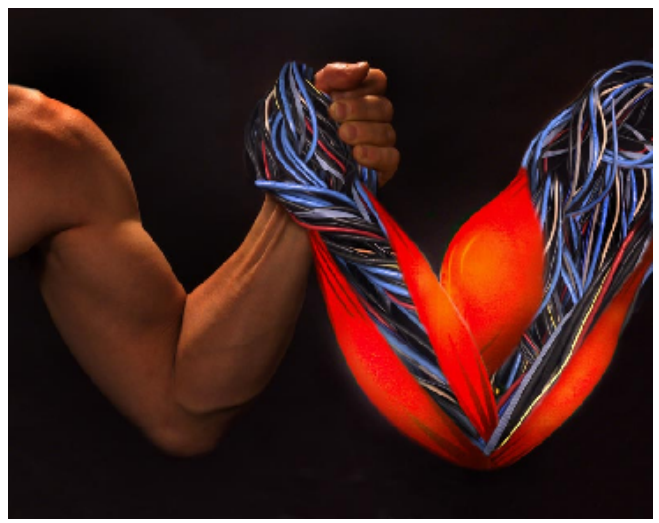

Figure 7: The icon of the arm-wrestling challenge for artificial muscles matched against a human opponent. 


\section{The current capabilities of humanlike robots}

Humanlike robots today have impressive capabilities in terms of emulating life-like human performance. Even though their performance is impressive, current humanlike robots are still unable to match the capability of humans as portrayed in science-fiction books and films. Even though they are portrayed to have superior capabilities that the current reality, science-fiction is inspiring and guiding innovation in the development of these technologies, as well as alert us of the potential dangers and negative possibilities they may hold [Kerman, 1991]. These possibilities include acting as an imposer and performing criminal acts. Some of the most recent humanlike robotic capabilities include robotic selfimprovement following their production, since robots can self-learn and obtain periodic updates. The sophistication of these robots that are being developed includes fully autonomous operation and self-diagnostics. In the future, they may be designed to travel on their own to a selected maintenance facility and receive periodic check-ups and repairs. In case of damage, future robots might be constructed from biomimetic materials that are capable of self-healing.

Within the currently limited intelligent capabilities of humanlike robots, they can be used for various practical applications. The least complex approach is to control them in a telepresence form, where they are made to perform highly sophisticated functions that are human-controlled in an avatar form. An example of such a form of control and operation is the Robonaut I (robotic astronaut) developed in the 1990s at NASA Johnson Space Center (JSC), Houston, Texas, USA. This robot mirrors the physical movements of the upper section of the human body; a photo of the Robonaut performing a task is shown in Figure 8. In recent years, the control was shifted from telepresence to fully autonomous operation. The original objective for its development was to operate as a cooperative robot that would perform functions outside the space shuttle or space station. Later, its applications were expanded to potential military and industrial tasks.

The progress in developing human-like robots is rapidly advancing but there are still many issues that are limiting their widespread use. These include their limited functions, relatively short battery charge and high cost. However, once human-like robots reach levels of mass production they are expected to become affordable and common household helpers that perform human-related services.

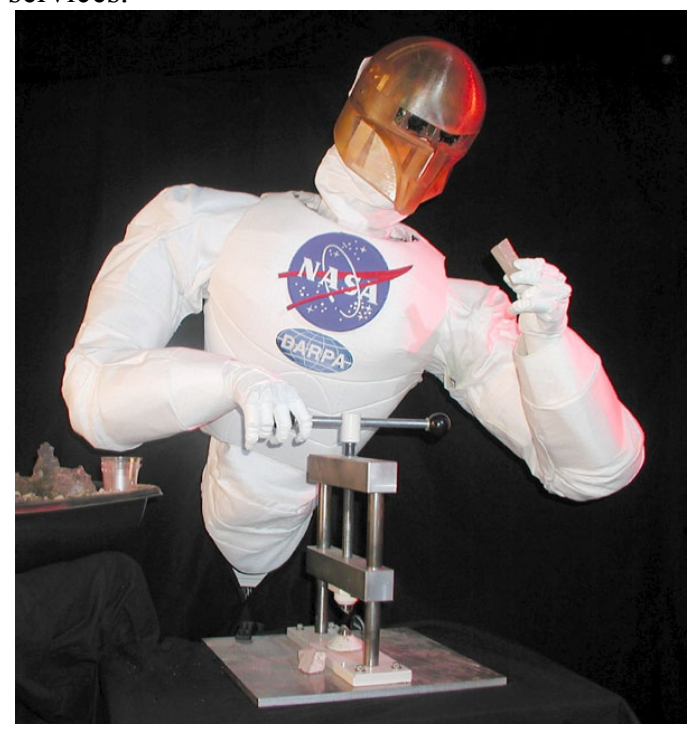

Figure 8: The Robonaut I is remotely controlled to perform physical tasks by mirroring the actions of a human (image: NASA Johnson Space Center).

\section{The DARPA challenge as indicator of the state-of-the-art}

In an effort to accelerate the development of humanoid robots, DARPA has initiated a challenge intended to lead to advances in humanitarian and disaster relief. The DARPA Robotics Challenge (DRC) consisted of requirements for extending aid to victims of natural or human-made disasters and conducting evacuation operations. Due to grave risks to the health and wellbeing of rescue and aid workers, some disasters have been found to be too large in scale or scope for delivering timely and effective human response. By setting up this challenge, DARPA sought to address the capability gap by promoting innovation in robotics for disaster-response operations, where robots will be made capable of executing complex tasks in dangerous environments. The focus of the challenge has been on the ability of robots to use tools and equipment that are commonly available in human environments. Advances were sought in terms of having robots operate autonomously with perception and decision-making abilities, dexterity, strength and endurance. 
Addressing the challenges required the development of hardware and software, with a secondary goal of making this development accessible to a broad number of users in order to help lowering the cost of robotic systems, while at the same time increasing their capabilities. The competitions that were held in 2013 and 2015 showed that the technology has a long way to go before such robots become operational in effective ways.

\section{Conclusions}

Humanlike robots are still far from replicating the full capabilities of real people. Current robots can perform only a limited number of functions and primarily execute specific tasks. Using AI, humanlike robots are capable of interpreting facial expressions and have personalized behavior that varies between the duplicates of particular robots. Some robots can walk or dance similarly to humans. However, many basic human tasks are still beyond current robots' capabilities. These include conducting a comprehensive conversation with a human on a broad range of subjects, walking fast in a crowd without bumping into anyone and operating for extended time without requiring a recharge. The process of overcoming these challenges related to the capabilities of human-like robots is evolutionary. Lowering the cost of producing humanlike robots requires the availability of standard hardware components and software platforms that are interchangeable and compatible.

Making humanlike robot is a multidisciplinary task requiring expertise in such fields as electromechanical engineering, computational and material sciences, neuroscience and biomechanics. Advances in artificial intelligence, effective actuators, artificial vision, speech synthesizers and recognition, mobility, control and many other fields are significantly contributing to making robots act more humanlike. The ability of future robots to conduct a comprehensive conversation will require the capability to recognize and "understand" more words than they can today, with significantly higher accuracy of interpreting text and verbal communication. As opposed to genetic cloning, which requires growth according to biological timescales, humanlike robots can be produced at the speed of manufacturing processes. As the rapid prototyping fabrication of humanlike figures or models made by computer graphics becomes easy to produce by a 3D printer, our neighborhoods may one day be filled with such robots. These may have the benefit of significantly helping humans, but will also potentially pose a significant risk to humans as a species.

\section{Acknowledgement}

Some of the research reported in this manuscript was conducted at the Jet Propulsion Laboratory (JPL), California Institute of Technology, under contract with the National Aeronautics and Space Administration (NASA). The author would like to express his appreciation to Leon Alkalai, JPL/Caltech, for his support provided through the JPL's Blue Sky Program.

\section{References}

Asimov I., "Runaround" (originally published in 1942), reprinted in I Robot, (1942) pp. 33- 51.

Bar-Cohen Y. (Ed.), Biomimetics: Nature-Based Innovation, ISBN: 9781439834763, ISBN 10: 1439834768, CRC Press, Taylor \& Francis Group, Boca Raton, FL (2011), pp. 1-788.

Bar-Cohen Y. (Ed.), Electroactive Polymer (EAP) Actuators as Artificial Muscles - Reality, Potential and Challenges, 2nd Edition, ISBN 0-8194-5297-1, SPIE Press, Bellingham, Washington, Vol. PM136, (March 2004), pp. 1-765.

Bar-Cohen Y. and C. Breazeal (Eds.), Biologically-Inspired Intelligent Robots, SPIE Press, Bellingham, Washington, Vol. PM122, ISBN 0-8194-4872-9 (May 2003), pp. 1-393.

Bar-Cohen Y. and D. Hanson, The Coming Robot Revolution - Expectations and Fears About Emerging Intelligent, Humanlike Machines, Springer, New York, ISBN: 978-0-387-85348-2, (2009).

Bar-Cohen Y., (Ed.), Biomimetics - Biologically Inspired Technologies, CRC Press, Boca Raton, FL, ISBN 0849331633, (November 2005), pp. 1-527.

Bar-Cohen, Y., (Ed.), Proceedings of the SPIE's Electroactive Polymer Actuators and Devices Conf., $6^{\text {th }}$ Smart Structures and Materials Symposium, SPIE Proc. Vol. 3669, (1999), pp. 1-414.

Breazeal C., Designing Sociable Robots. MIT Press, Cambridge, MA (2004).

Dautenhahn K. and C. L. Nehaniv (Eds.), Imitation in Animals and Artifacts, ISBN-10: 0262042037, MIT Press, May 2002.

Full R. J. and K. Meijir, "Metrics of Natural Muscle Function,” Chapter 3 in [Bar-Cohen, 2004] pp. 73-89.

Harris G., "To Be Almost Human Or Not To Be, That Is The Question," Engineering Feature Article, (Feb 2007) pp. 37 38.

Kerman J. B. Retrofitting Blade Runner: Issues in Ridley Scott's Blade Runner and Philip K. Dick's Do Androids Dream of Electric Sheep?, Bowling Green, OH: Bowling Green State University Popular Press, ISBN 0-87972-509-5 (1991). 
Plantec P. M. and R. Kurzwell (Foreword), Virtual Humans: A Build-It-Yourself Kit, Complete With Software and StepBy-Step Instructions, ISBN-10: 0814472214, ISBN-13: 978-0814472217, AMACOM/American Management Association, (2003).

Rosheim M. Robot Evolution: The Development of Anthrobotics Wiley, (1994).

Rosheim, M. "Leonardo's Lost Robot," Journal of Leonardo Studies \& Bibliography of Vinciana, Vol. IX, Academia Leonardi Vinci (September 1996). Pp. 99-110.

Shelley M., Frankenstein, Lackington, Hughes, Harding, Mavor \& Jones, (1818).

Stoica A., Robot fostering techniques for sensory-motor development of humanoid robots, Robotics and Autonomous Systems 37 (2001) pp. 127-143.

Vincent J. F. V., "Stealing ideas from nature," Deployable Structures, S. Pellegrino (Ed), Springer, Vienna, (2005) pp. 51-58. 